\title{
FULLY NONLINEAR BOUSSINESQ-TYPE MODELLING OF INFRAGRAVITY WAVE TRANSFORMATION OVER A LOW-SLOPING BEACH
}

\author{
Marion Tissier ${ }^{1}$, Philippe Bonneton ${ }^{2}$, Gerben Ruessink ${ }^{1}$, Fabien Marche ${ }^{3}$, \\ Florent Chazel ${ }^{4}$ and David Lannes ${ }^{5}$
}

\begin{abstract}
Recent field studies over low sloping beaches have shown that infragravity waves could dissipate a significant part of their energy in the inner surf zone. This phenomenon and the associated short- and long-wave transformations are not well-understood. In this paper, we assess the ability of the fully nonlinear Boussinesq-type model introduced in Bonneton et al. (2011) to reproduce short and long wave transformation in a case involving a strong infragravity wave dissipation close to the shoreline. This validation study, based on van Dongeren et al. (2008)'s laboratory experiments, suggests that the model is able to predict infragravity wave breaking as well as the complex interactions between short and long waves in the surf zone.
\end{abstract}

Keywords: Infragravity wave dissipation; Fully Nonlinear Boussinesq-type model; Long-wave breaking; Bore merging;

\section{INTRODUCTION}

Very few studies have focused on wave transformation over low-sloping beaches. This type of beach, however, is characterized by complex non-linear dynamics. Low-sloping beaches are often high-energy beaches, where infragravity waves dominate the water motion in shallow water. However, contrary to relatively steep beaches where infragravity waves generally propagate shoreward and reflect to form a standing or quasi-standing wave pattern (Guza and Thornton, 1985), infragravity wave dissipation cannot be ignored in case of low-sloping beaches. Recent field studies over a 1:80 sloping beach (Ruessink et al., in press) have shown that the reflection coefficients could be less than 10-30\%, preventing the development of standing wave patterns and impacting the sediment transport significantly. Several mechanisms have been proposed in the literature to explain infragravity wave dissipation. Early studies suggested that this dissipation could result from the effects of bed friction (e.g., Herbers et al., 1995). Field data analysis by Henderson et al. (2006) and Thomson et al. (2006) showed that the observed energy loss was resulting from nonlinear energy transfer back to the short waves. Recently, van Dongeren et al. (2007) demonstrated that the breaking of the infragravity waves themselves could be an important dissipation mechanism for low values of the normalized bed slope $\beta$. These findings were supported by Ruessink et al.'s field study, who identified infragravity-wave breaking as the most likely dissipation mechanism in the inner surf zone of their very low-sloping beach. Recently, Ruju et al. (2012) suggested that, based on a numerical evaluation of the radiation stress, nonlinear energy transfer could be the leading dissipation mechanism in the outter surf zone, while infragravity-wave breaking could occur in the most inner part. However, the exact energy dissipation mechanisms and their relative importance remain unclear. Numerical modeling could be an efficient way to investigate further these questions.

In this paper, we present a numerical study of short and long-wave transformation in the surf zone of a low-sloping beach. This study will be performed using the Fully Nonlinear Boussinesq-type model introduced in Bonneton et al. (2011) and Tissier et al. (2012). More precisely, we investigate the ability of our numerical model to predict wave dynamics for a case involving a strong infragravity-wave dissipation. This validation study is based on van Noorloos (2003) bichromatic wave experiments, analyzed in Van Dongeren et al. (2007). Our numerical model is first introduced. Detailed comparisons between experimental and numerical results are then performed. We finally present some preliminary analysis concerning short and long-wave interactions when approaching the shoreline.

\section{NUMERICAL MODEL}

In this section we briefly present the main characteristics of our numerical model SURF-GN. The reader is referred to Bonneton et al. (2011) and Tissier et al. (2012) for further details.

\footnotetext{
${ }^{1}$ Faculty of Geosciences, Utrecht University, P.O. box 80115, 3508 TC Utrecht, The Netherlands

2 UMR CNRS 5805 EPOC - Université Bordeaux 1, Avenue des Facultés - 33405 Talence Cedex - France

${ }^{3}$ Université de Montpellier 2, UMR CNRS 5149, Place Eugène Bataillon, 34095 Montpellier, France

4 INSA - Département GMM, 135 avenue de Rangueil, F-31077 Toulouse Cedex 4, France

${ }^{5}$ DMA/CNRS, Ecole Normale Supérieure, 45 rue d'UIm, 75005 Paris, France
} 


\section{Governing Equations}

The model is based on the Serre Green-Naghdi (S-GN) equations, considered as the basic fully nonlinear weakly dispersive equations (Lannes and Bonneton, 2009). These equations can be formulated in terms of the conservative variables (h, hv) in the following nondimensionalized form (see Bonneton et al., 2011):

$$
\left\{\begin{array}{l}
\partial_{t} h+\varepsilon \nabla \cdot(h v)=0 \\
\partial_{t}(h v)+g h \nabla \zeta+\varepsilon \nabla \cdot(h v \quad v)=-\mu D,
\end{array}\right.
$$

where $\zeta$ is the surface elevation, $h=1+\varepsilon \zeta-b$ the water depth, $b$ the variation of the bottom topography and $\mathbf{v}=(u, v)$ the depth averaged velocity. $\varepsilon=H / h 0$ is the non-linear parameter with $H$ the wave height and $h 0$ the typical water depth, and $\mu=(h 0 / \lambda)^{2}$, with $\lambda$ the wave length. $D$ characterizes non-hydrostatic and dispersive effects, and has been written such as it does not require the computation of any third order derivative. It is noteworthy that if $D=0$, we obtain the Nonlinear Shallow Water Equations (NSWE) in their conservative form. The formulation is therefore well-suited for a splitting approach separating the hyperbolic (NSW) and dispersive parts of the equations, allowing an easy coupling of the sets of equations.

\section{Numerical Strategy}

At each time step dt, we decompose the solution operator $\mathrm{S}(\bullet)$ associated to the equations (1) using the following second order splitting scheme:

$$
\mathrm{S}(\mathrm{dt})=\mathrm{S} 1(\mathrm{dt} / 2) \mathrm{S} 2(\mathrm{dt}) \mathrm{S} 1(\mathrm{dt} / 2)
$$

where S1 and S2 are associated to the hyperbolic and dispersive parts of the S-GN equations, respectively. S1 is treated using a finite-volume method, while S2 is treated using a classical finitedifference method.

The numerical methods used in S1 are those developed in the extensively-validated NSW code SURF-WB (Marche et al., 2007, Berthon and Marche, 2008). It is based on high-order positive preserving well-balanced shock-capturing schemes. They are able to handle breaking bore propagation without any parameterization of the energy dissipation, as well as moving shorelines without any tracking method.

\section{Wave Breaking}

When the wave is ready to break, we switch locally from S-GN equations to NSWE by skipping the step S2 in the vicinity of the front. Energy dissipation due to wave breaking is then predicted by the shock theory, and no complex ad-hoc parameterization is required. More precisely, we locate the wave fronts at each time step and then determine if they are breaking or not based on the amount of energy dissipated in their vicinity (see Tissier et al. 2012 for details). We then apply criteria for the initiation or termination of breaking based respectively on a critical front slope $\left(\Phi_{c}=30^{\circ}\right)$ and a critical Froude number $\left(F r_{c}=1.3\right)$.

\section{RESULTS}

\section{Numerical Set-up}

In this paper, we focus on the experiment A-1 analyzed in van Dongeren et al. 2007, where a very low reflection coefficient $(\mathrm{R} \sim 0.1)$ of the infragravity waves was observed at the shoreline. Bichromatic waves of primary frequency $f_{1}=0.6714 \mathrm{~Hz}$ and $f_{2}=0.4761 \mathrm{~Hz}$, and amplitudes $a_{1}=0.06 \mathrm{~m} a_{2}=0.012 \mathrm{~m}$ were generated on the horizontal part of the beach and were then propagating and breaking over a 1:35 sloping beach.

Our numerical domain is presented in Figure 1. The waves were generated at the offshore boundary according to the linear theory with a generating-absorbing boundary condition. A quadratic friction term was introduced with a non-dimensional friction coefficient $C_{f}=0.005$. A space grid of $d x=0.035$ $\mathrm{m}$ and a time step of $d t=0.01 \mathrm{~s}$ are considered for this simulation.

During the experiments, wave gauges were deployed with a high resolution along the flume $(50 \mathrm{~cm}$ in the shoaling zone and $30 \mathrm{~cm}$ in the surf zone). However, as we are mainly interested in short and long wave dynamics in the surf zone, we limit our model/data comparisons to the locations presented in Figure 1. 


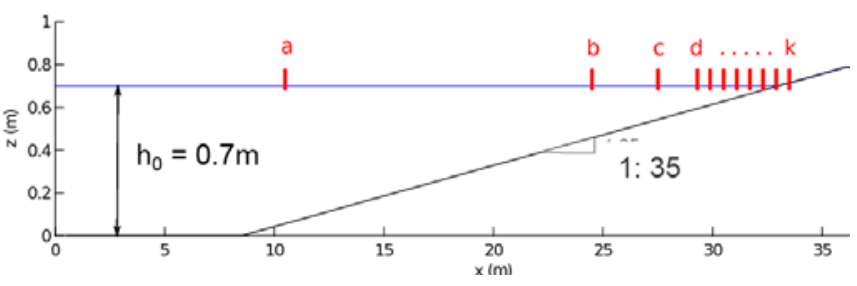

Figure 1. Numerical domain and positions of the selected wave gauges (red vertical lines). Breaking starts just after the wave gauge $b$.

\section{Nonlinear Wave Transformation}

Figure 2 compares experimental and numerical time-series of free-surface elevation at the locations introduced in Figure 1. Despite a slight underestimation of the wave height, in particular in the last stages of shoaling (Fig. 2b), a fairly good agreement with the experimental data is obtained before breaking.

The agreement between model and data remains good in the surf zone, despite an increasingly complex nonlinear behavior. Wave dynamics is indeed mainly dominated by the short waves at the most outer surf zone locations (Fig. 2c,d), and becomes increasingly long-wave dominated during the propagation shoreward. In the inner surf zone (Fig. 2, e to k), strong variations of the mean water-level can be observed, associated with a modulation of the short-wave periods. Successive bore-merging processes can be seen (e.g., Fig. 2 red dashed lines), leading to an increase of the period of the breaking waves when approaching the swash zone (Fig. 2 j,k). These processes, well-described by the model, will be analyzed further in the next section.

The infragravity part of the signal, defined as the component of frequency $\Delta \mathrm{f}=\mathrm{f}_{1}-\mathrm{f}_{2}$ and its higher harmonics (see van Dongeren et al., 2007) is more specifically studied in Figure 3. The main discrepancies between model and data are observed at the most offshore locations, but the agreement increases significantly in the surf zone. The infragravity wave is first steepening while gaining in amplitude (c to i). Its amplitude then starts to decrease ( $\mathrm{j}$ to $\mathrm{k}$ ), while its shape evolves toward a sawtooth profile. These transformations, resembling the behavior of typical inner surf-zone waves, has been identified by van Dongeren et al. (2007) as infragravity-wave breaking.

The nonlinear transformations of the IG wave are characterized further in Figure 4, which shows the cross-shore evolution of the wave skewness $(S k)$ and the asymmetry $(A s)$ in the surf zone. These wave shape parameters are computed in the following way:

$$
S k=\frac{\left\langle\left(\zeta_{I G}-\bar{\zeta}_{I G}\right)^{3}\right\rangle}{\left\langle\left(\zeta_{I G}-\bar{\zeta}_{I G}\right)^{2}\right\rangle^{3 / 2}}, \quad A s=-\frac{\left\langle H\left(\zeta_{I G}-\bar{\zeta}_{I G}\right)^{3}\right\rangle}{\left\langle\left(\zeta_{I G}-\bar{\zeta}_{I G}\right)^{2}\right\rangle^{3 / 2}},
$$

where $\xi_{\text {IG }}$ is the free-surface elevation of the infragravity wave, \langle\rangle the time-average operator and $H$ the Hilbert transform. A strong increase of the wave asymmetry can be observed in the inner surf zone, corresponding to the steepening of the infragravity wave. Close to the swash zone, the asymmetry is close to 1.5 while the skewness is close to zero. These values are similar to the typical skewness and asymmetry observed for (short) surf-zone waves. It is worth noting that a similar infragravity wave steepening and amplitude decrease has been observed for simulations without friction (not shown here). It supports the idea that friction does not play a leading role in the observed infragravity wave transformation.

Finally, Figure 5 shows the spatial variation of the magnitude and phase of the long-wave component $\Delta$ f. No clear nodal structure is observed for the amplitude. A general increase (despite some oscillations) is first observed, followed by a strong decrease when approaching the swash zone due to infragravity-wave breaking. This indicates the development of a progressive pattern, indicating a low reflection of the long wave at the shoreline, and therefore a strong infragravity-wave dissipation. This is supported by the quasi-linear increase of the long-wave phase, in opposition with the 180-degree phase jumps at the nodes expected for a standing wave. The decrease of the long-wave amplitude due to breaking is well-predicted by the numerical model. This suggests that SURF-GN reproduces correctly the dissipation mechanisms in very shallow water. 


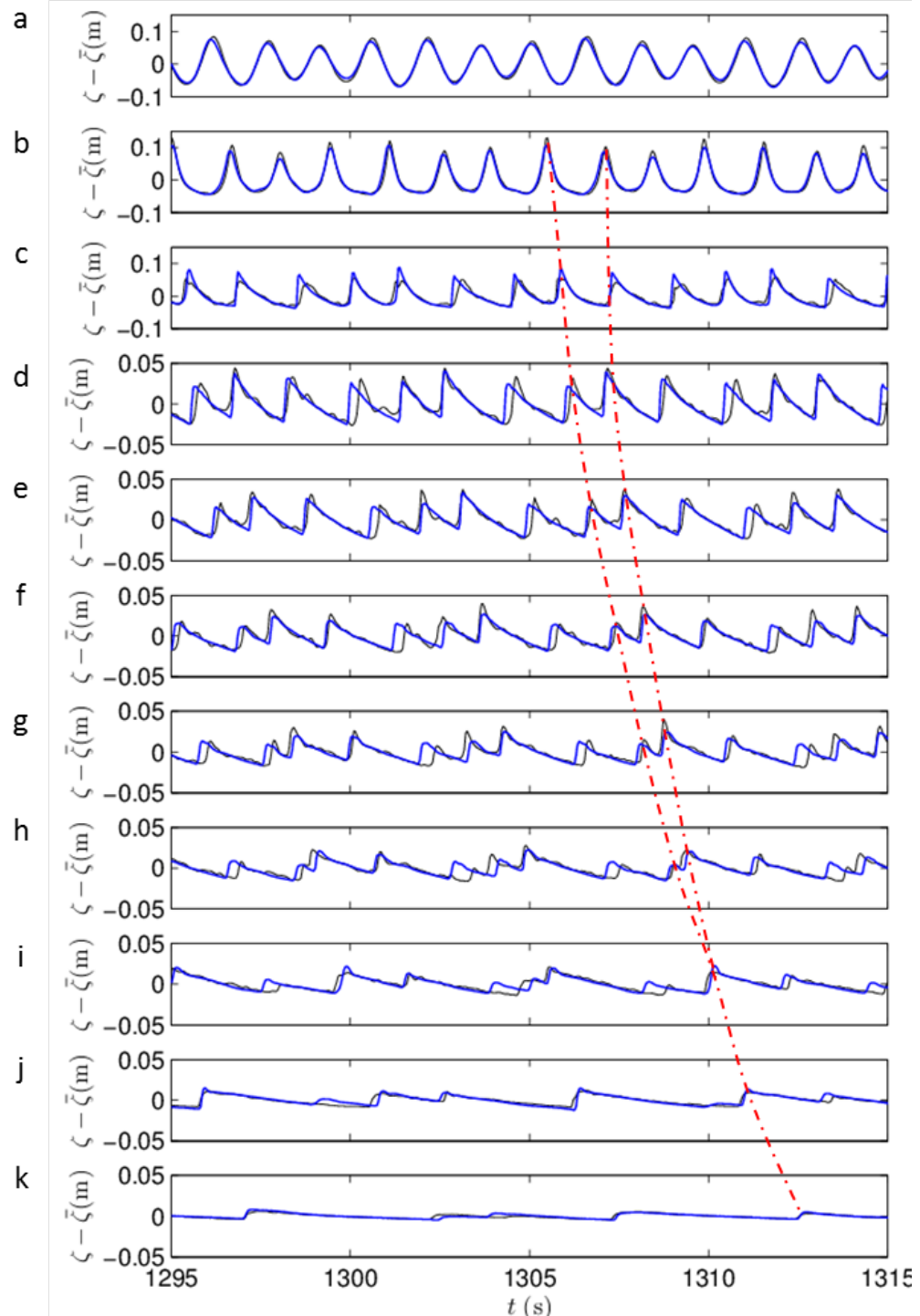

Figure 2. Timeseries of free-surface elevation relative to the mean water level at the wave gauges a to $k$ (see Fig. 1). Blue thick lines : model results. Black lines : experimental data (Case A1, van Dongeren et al. 2007). 


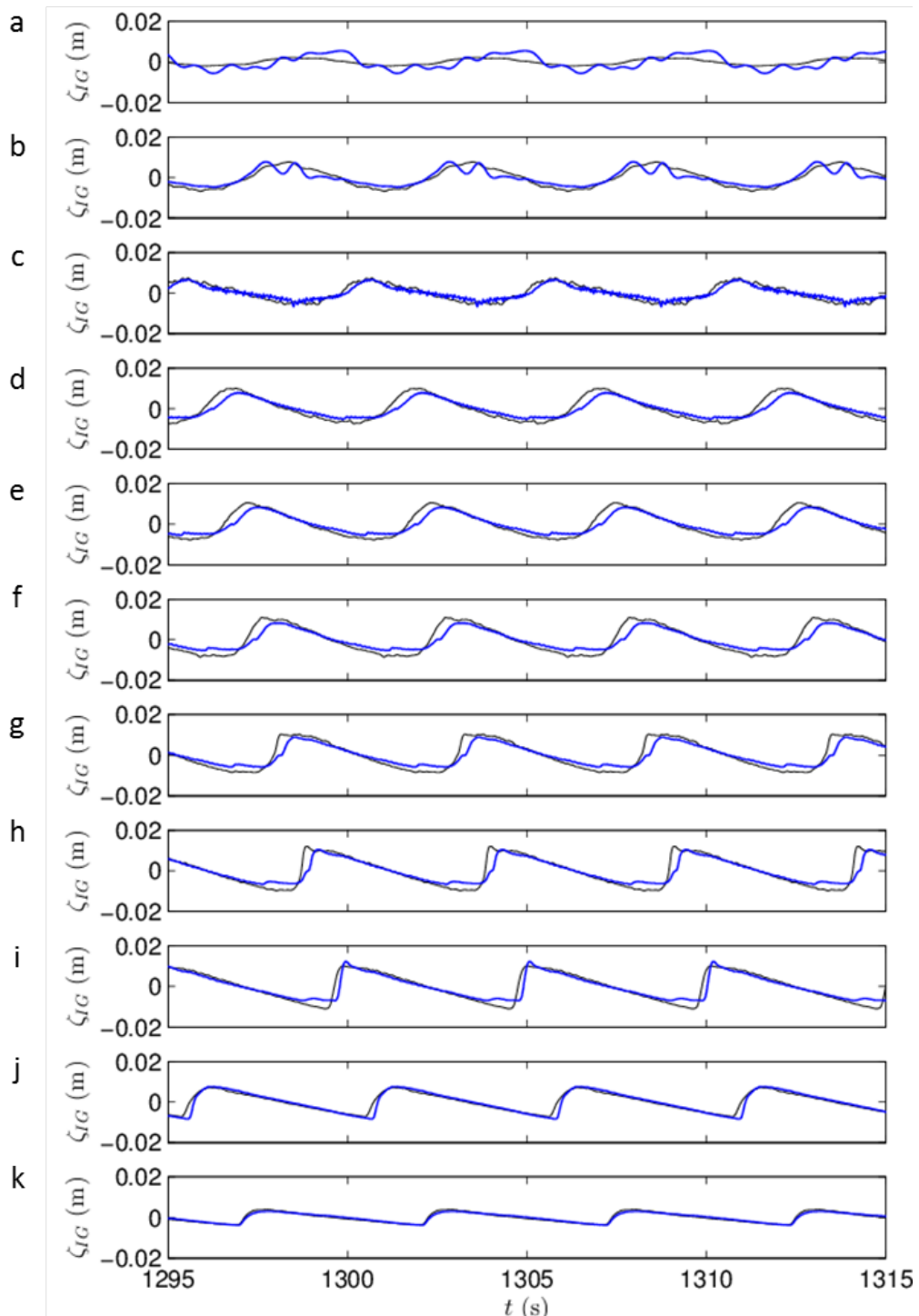

Figure 3. Timeseries of IG wave signal (low-frequency component $\Delta f=f_{1}-f_{2}$ and its higher harmonics) at the wave gauges a to $\mathrm{k}$ (see Fig. 1). Blue lines : model results. Black lines : experimental data (Case A1, van Dongeren et al. 2007).
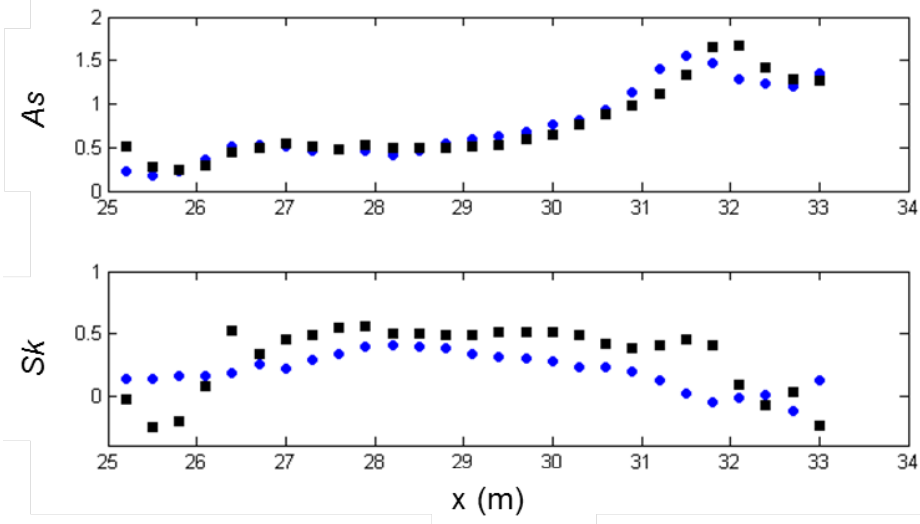

Figure 4. Spatial evolution of infragravity wave skewness (Sk) and asymmetry (As) across the (short wave) surf zone. Blue dots : computed from the numerical data. Black squares : computed from the experimental data. 

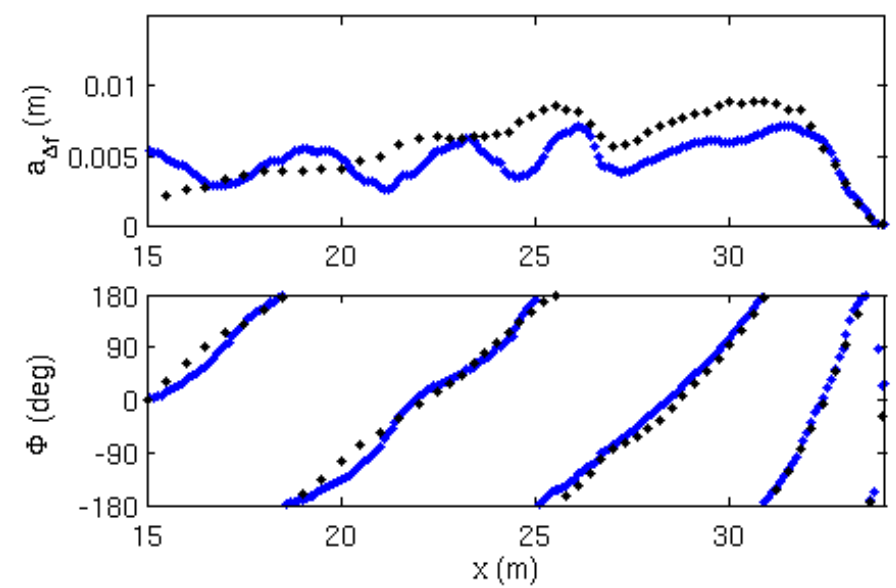

Figure 5. Spatial structure of the long wave component $\Delta \mathrm{f}$. Top panel : magnitude of the $\Delta \mathrm{f}$-component, Bottom panel : phase difference relative to $x=15 \mathrm{~m}$. Blue dots : computed from the numerical data. Black squares : computed from the experimental data.

\section{Short/Long Wave Interaction}

In the inner surf zone, both short and long waves exhibit a highly non-linear behavior, and will interact in a complex way. The effect of the infragravity wave transformations on short-wave dynamics is studied more specifically in this section. Figure 6 presents a time-space diagram of the free-surface elevation computed with the model. A strong modulation of the celerity field can be observed for $x>30$ $\mathrm{m}$, leading to the merging of the breaking bores (see red boxes, Fig. 6). Figure 7 highlights the leading role of the infragravity wave in these transformations. Both the water elevation and velocity fields are represented at three times, before (b,c) and after (d) merging. The waves located on the crest of the long wave (e.g. wave A, Fig 7, b) propagate in deeper water and benefit from the positive current associated with the long wave. They will therefore propagate faster than the waves located in the trough, such as wave $\mathrm{C}$, which combine a smaller water depth and a negative long-wave current (see Fig.7,c). This leads to the convergence of the short waves on the long wave front described in van Dongeren et al. (2007). The waves on the crest progressively catch up with the infragravity wave front, while the waves on the trough are captured by it. This happens to such an extent that, very close to the shoreline, the long wave front coincides with the roller previously associated with the short waves. The infragravity wave is then breaking.

\section{CONCLUSIONS AND PERSPECTIVES}

In this paper we have shown that our fully nonlinear Boussinesq-type model was able to reproduce accurately both short and long-wave transformations in the surf zone of a relatively low-sloping beach. In particular, the model can predict accurately the steepening and breaking of the infragravity waves, without any specific calibration. It is worth noting that this test-case is very challenging for Boussinesqtype models in general. Indeed, most of them include an ad-hoc parameterization of the dissipation due to wave breaking specifically developed and calibrated for the description of short wave components. More validations using van Noorloos (2003)'experiments should be performed to assess the model ability to describe more or less dissipative cases for the infragravity waves. This work is in progress and showed promising results, suggesting that the model can handle the main mechanisms driving infragravity wave transformation in the surf zone. The model could then be used as a tool to understand better infragravity wave dynamics and dissipation over low sloping beaches. In particular, the relative importance of the different dissipation mechanisms could be assessed through the computation of the energy balance for the IG waves (e.g. Henderson et al., 2006, Ruju et al. 2012). However, it should be noted that the methodology developed by Henderson et al. (2006) assumes weakly nonlinear infragravity waves, and that their amplitude is much smaller than the short waves. These conditions are not satisfied in our case, in particular just seaward of the swash zone, where the nonlinear parameter is of order 1 for the IG waves. Alternative approaches, such as the one presented in Ruju et al. (2012), could therefore be more adapted to analyze further our numerical results. 


\section{ACKNOWLEDGMENTS}

The authors would like to thank Job van Noorloos and Ap van Dongeren for providing the experimental data. This work has been supported by the ANR MathOcean, the project ECOSCONYCIT and the ANR MISEEVA.

\section{REFERENCES}

Ruessink, B.G., Boers, M., van Geer P.F.C., de Bakker, A.T.M., Pieterse, A., Grasso, F. and de Winter, R.C., in press. Towards a process-based model to predict dune erosion along the Dutch Wadden coast. Netherlands Journal of Geosciences.

Berthon, C. and F. Marche, 2008. A positive preserving high order VFRoe scheme for shallow water equations: A class of relaxation schemes, SIAM Journal of Scientific Computing, 30(5), 25872612.

Bonneton, P., Chazel, F., Lannes, D., Marche, F., Tissier, M., 2011. A splitting approach for the fully nonlinear and weakly dispersive Green-Naghdi model, Journal of Computational Physics 230, 1479-1498.

van Dongeren, A., Battjes, J., Janssen, T., van Noorloos, J., Steenhauer, K., Steenbergen, G., Reniers, A., 2007. Shoaling and shoreline dissipation of low-frequency waves, Journal of Geophysical Research 112. C02011.

Guza, R.T., Thornton, E.B., 1985. Observations of surf beat, Journal of Geophysical Research 87, 483-491.

Herbers, T.H.C., Elgar, S., Guza, R., 1995. Generation and propagation of infragravity waves, Journal of Geophysical Research 100, 24863-24872.

Henderson, S.M., Guza, R.T., Elgar, S., Herbers, T.H.C., Bowen, A.J., 2006. Nonlinear generation and loss of infragravity wave energy, Journal of Geophysical Research 111, C12007.

Lannes, D., and P. Bonneton. 2009. Derivation of asymptotic two-dimensional time-dependent equations for surface water wave propagation, Physics of Fluids, 21(1).

Marche, M., P. Bonneton, P. Fabrie and N. Seguin. 2007. Evaluation of well-balanced bore-capturing schemes for 2D wetting and drying processes, Int. J. Num. Meth. Fluids, 53(5), 867-894.

van Noorloos, J., 2003. Energy transfer between short wave groups and bound long waves on a plane slope, Master thesis, TU Delft, The Netherlands.

Ruju, A., Lara, J., Losada, I., 2012. Radiation stress and low-frequency energy balance within the surf zone: A numerical approach, Coastal Engineering 68, 44-55.

Thomson, J., Elgar, S., Raubenheimer, B., Herbers, T.H.C., Guza, R.T., 2006. Tidal modulation of infragravity waves via nonlinear energy losses in the surfzone, Geophysical Research Letters 33.

Tissier, M., Marche, F., Bonneton, P., Chazel, F., Lannes, D., 2012. A new approach to handle wave breaking in fully nonlinear Boussinesq-type model, Coastal Engineering, 67, 54-66. 


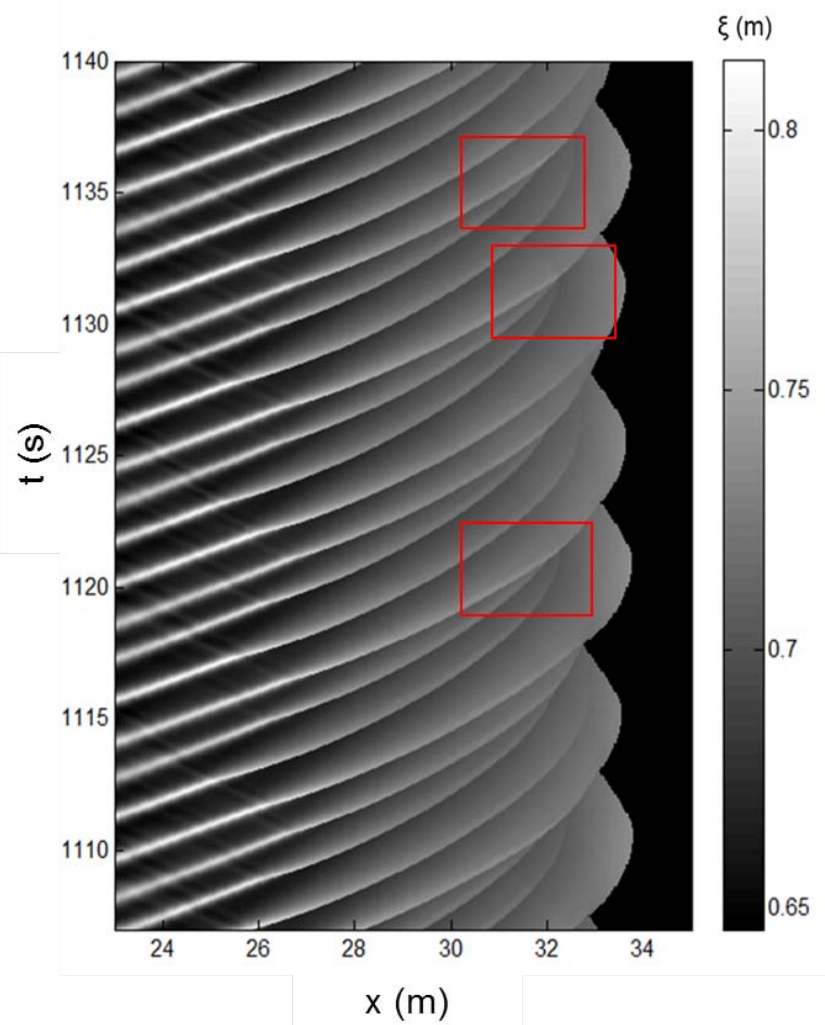

Figure 6. Timestacks of the free-surface elevation predicted by the model. Black area on the right : dry beach.

(a)
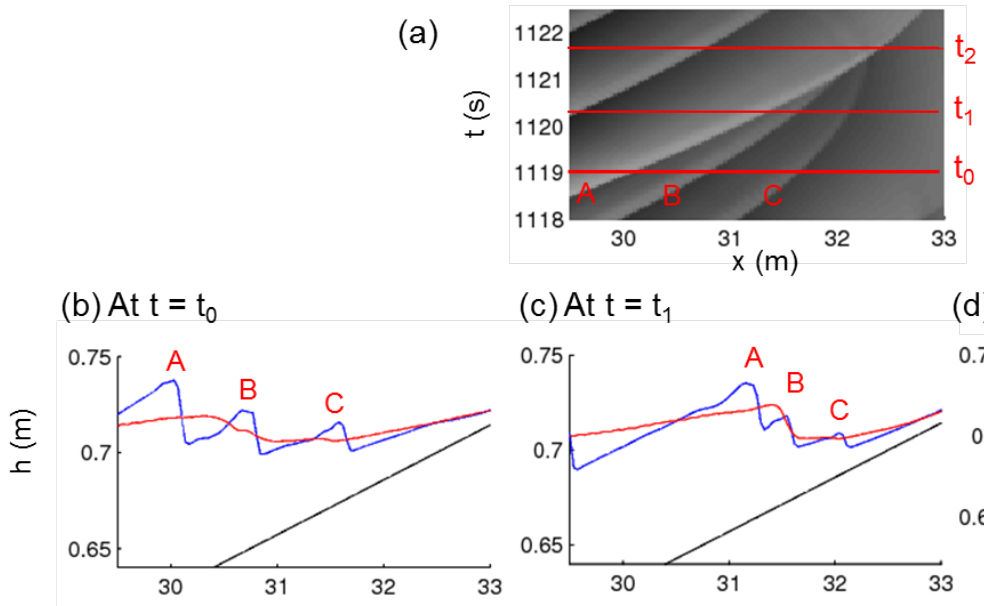

(d) At $t=t_{2}$
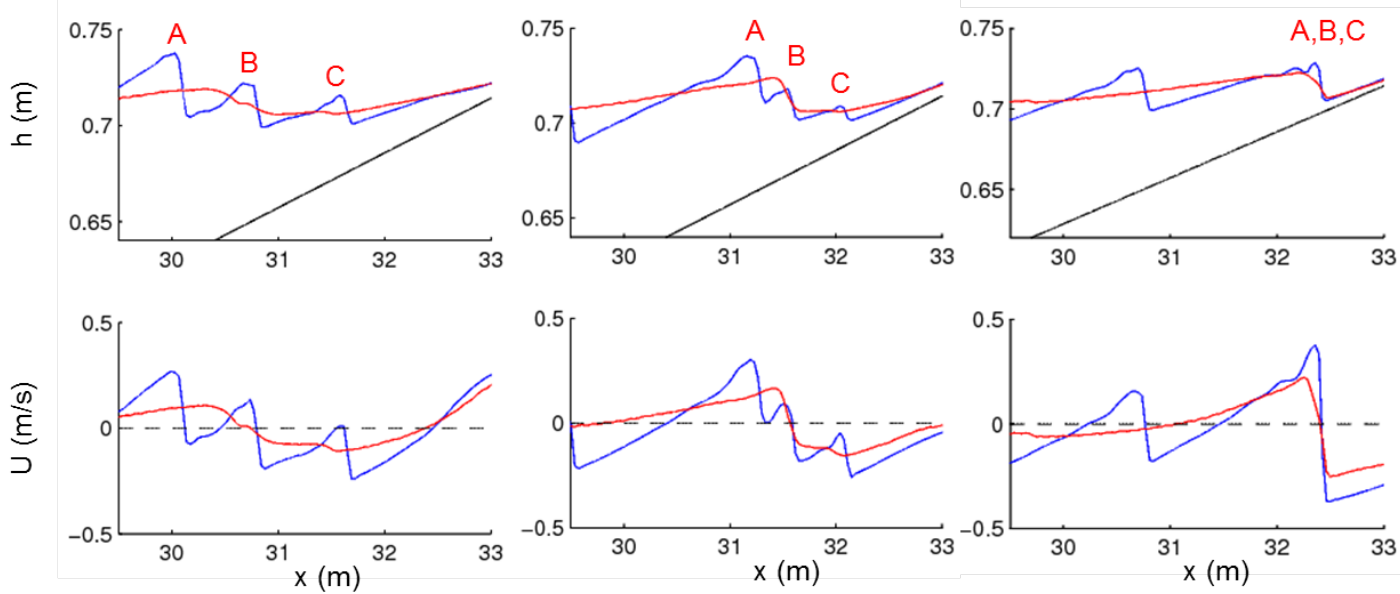

Figure 7. Short/long wave interaction and bore merging. (a) Zoom on a bore merging. (b) to (d) surface elevation and velocity fields before (b and $c$ ) and after the merging (d). In blue: non-filtered signal. In red : IG signal. 